\title{
Research and practice of laser cutting machine adapting to the special processing requirements
}

\author{
Chen Yun-xin
}

(School of Electromechanical \&Architectural Engineering, Jianghan University, Wuhan, 430056, China)

Keywords: Laser cutting machine; ANSYS; Large arm of machine tool; dynamic analysis

\begin{abstract}
Analysis by finite element method of laser cutting machine arm static and dynamic, theoretically study the static and dynamic characteristics, and the analysis results are used to correct the finite element model, which can get the accurate model with structural parameters, and then modify the structure, uniform and reasonable distribution of the load, improve the utilization rate of materials, reduce the production cost so, the structure and properties of the laser cutting machine to achieve the optimal arm; and then through the analysis of laser focus effect on the precision of laser cutting, laser cutting speed and cutting precision of the influence of laser, different materials processed material influence on the precision of laser cutting, laser processing needs specific conclusion to adapt to the cutting machine if the laser cutting machine used properly, so we can get a high productivity and economy..
\end{abstract}

\section{Introduction}

The rapid development of our national economy, the needs of all walks of life more and more on the laser cutting machine, also put forward higher requirements on the accuracy of laser cutting machine, domestic and international market competition is very intense. Many laser cutting machine tool manufacturers in the world focus on the development of high speed, high precision, high efficiency, high efficiency of the machine tool. China's laser cutting machine design for a long time still use the experience and analogy of traditional design method, design the machine not only poor performance, heavy structure, speed and accuracy is not high, and the long design cycle, high cost, slow renewal. With the combination of electronic technology, computer technology and machine tool technology analysis, we introduce the modern design ideas and methods, calculated by the finite element method of static and dynamic characteristics, analysis to optimize the laser cutting machine, realize the real meaning of the design, to ensure low power, low consumption, low cost, high efficiency and high the advantages of high precision and efficiency. The large arm of the laser cutting machine tool is driven by the laser cutting head for processing, and the structural characteristics will directly affect the machining precision and quality. Laser cutting machine tool large arm structure requires both small size, light weight, and requires a high static and dynamic characteristics, so that it can work safely and reliably, to obtain high precision, high efficiency. Therefore, it is necessary to make a detailed analysis of the structure and dynamic characteristics of the large arm of the laser cutting machine tool and optimize the design, so that the limited material can be used to maximize its performance. In order to analyze the specific processing requirements of laser cutting machine to better study, must focus on the laser cutting speed position influence on precision of laser cutting, laser effect on the precision of laser cutting of different materials affected by processing materials on the precision of laser cutting for analysis.

In the late 1960s, with the development of computer technology, the finite element method was applied to engineering practice. As a kind of numerical calculation method of finite element method, it has appeared many outstanding advantages of CAE software and on this basis (such as finite element analysis developed by American ANSYS (FEA) software ${ }^{[1]}$; MSc MSC.Nastran and MSC.Patran etc.), realizes the calculation of combined machine technology and finite element theory, can greatly improve the efficiency and quality of the design of machine tool structure. At present, using the finite element method to establish the static and dynamic model of the machine 
tool structure has become a widely used method in the theoretical modeling of machine tools.

\section{Mechanical characteristics analysis of the large arm of laser cutting machine tool:}

The solid model of the big arm is shown in Figure 1. Figure 2 shows the shape of the deformation of the large arm. The node displacement is shown in Figure 3. the Von Mises distribution of the stress distribution is shown in Figure 4.

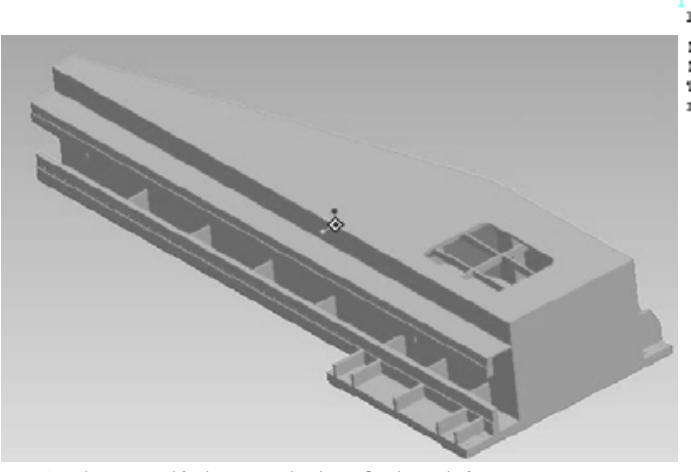

Fig. 1 the solid model of the big arm

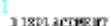

2 irep-1

IIS -1

TiE $2=1$
AN
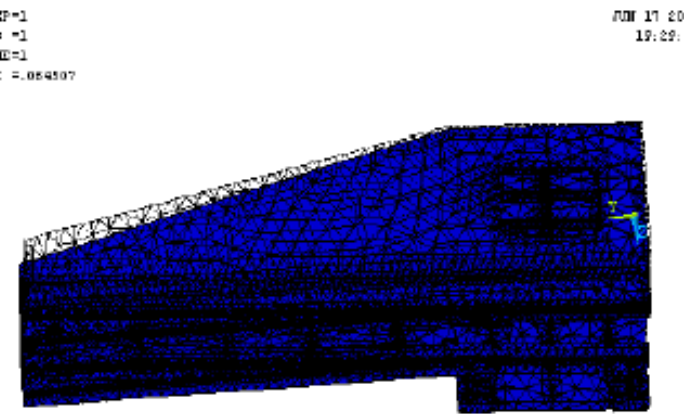

Fig. 2 deformation shapes of the big arms

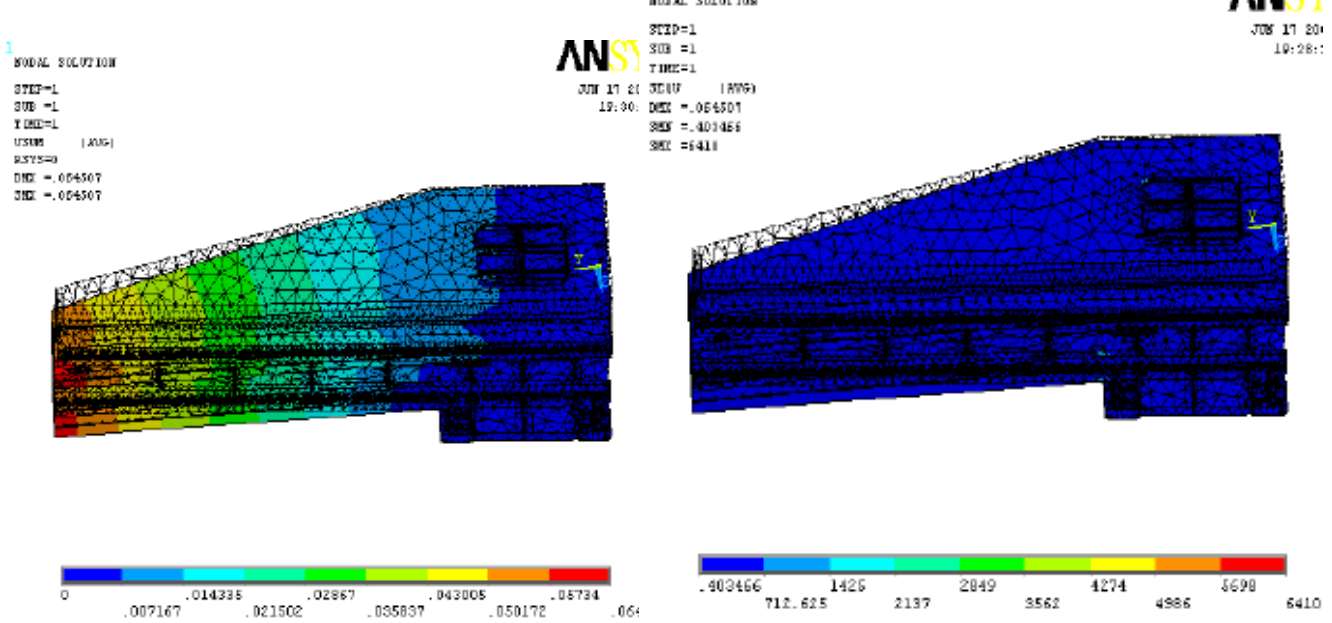

Fig. 3 the node displacement ${ }^{.035037}$ Fig. 4 the Von Mises distribution of the stress distribution

From the above we can see that the big arm farther from the bed in the distance, the greater the deformation. The allowable stress $112000 \mathrm{KPa}$ material Aluminum Alloy arm, the arm of the equivalent maximum stress is far less than the allowable stress value, material properties are not fully realized, which belongs to the design, resulting in the waste of materials, for the rational use of materials, reduce manufacturing cost, it is necessary to arm structure optimization design.

The relative displacement of the first four modes of the large arms and each of the modes of vibration are shown in the following diagram:
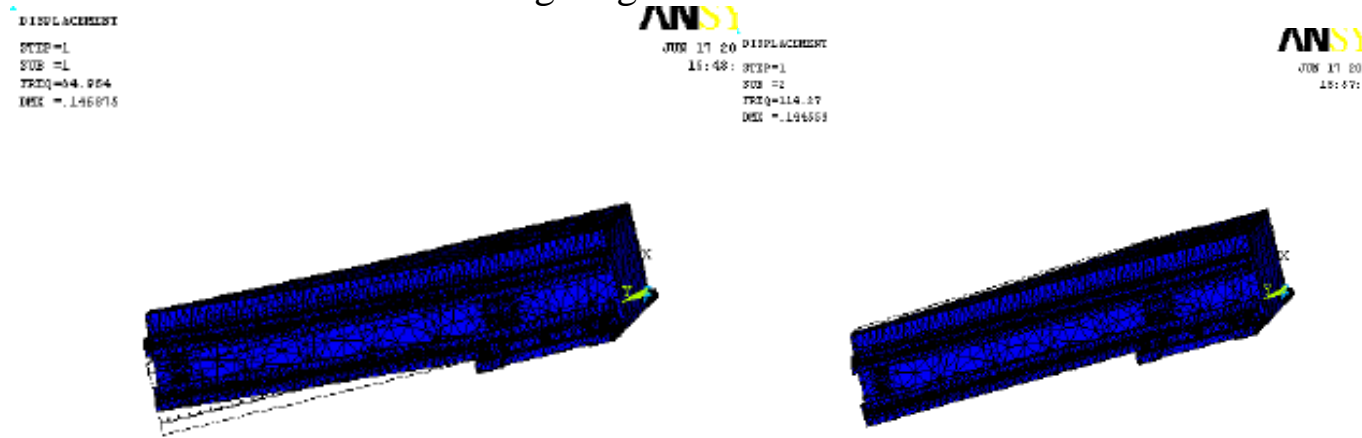

Fig. 5 first order vibration mode of the big arms Fig. 6 second order vibration mode of the big arms 


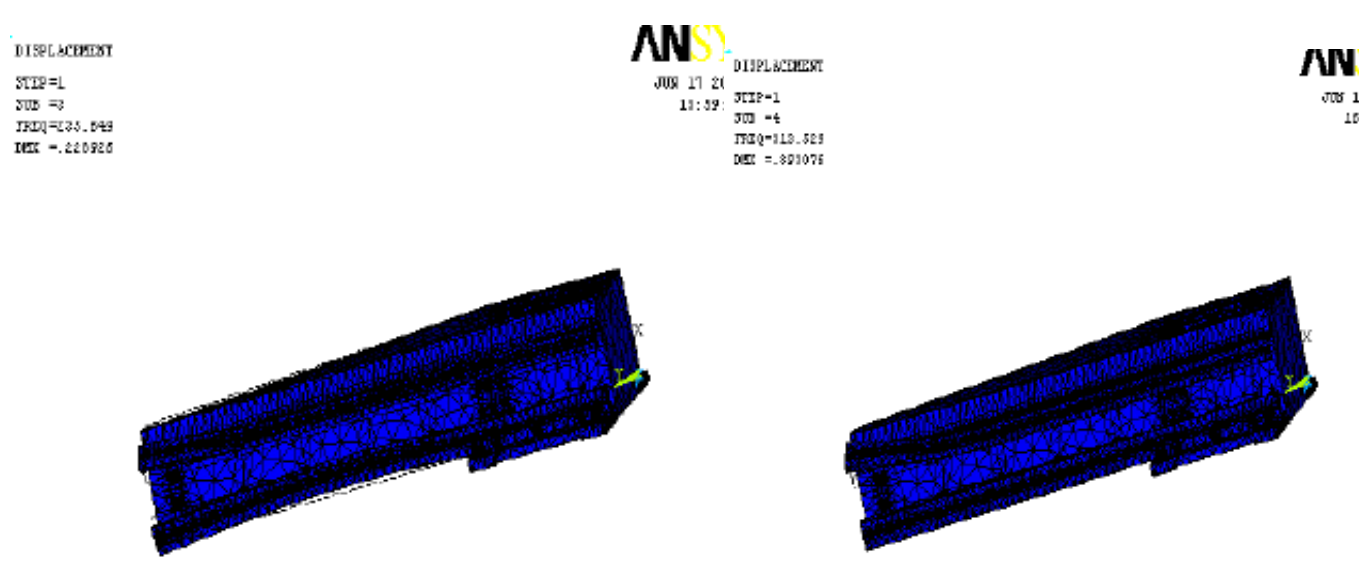

Fig. 7 third order vibration mode of the big arms Fig. 8 fourth order vibration mode of the big arms

The modal natural frequencies and the maximum relative displacement of the large arms are shown in table 1.

Table 1 modal analysis results of large arms

\begin{tabular}{|c|c|c|}
\hline Modal order & natural frequency $/ \mathrm{Hz}$ & Maximum vibration relative position $/ \mathrm{mm}$ \\
\hline first order & 84.964 & 0.146875 \\
\hline second order & 114.27 & 0.144559 \\
\hline third order & 235.65 & 0.228926 \\
\hline fourth order & 313.53 & 0.893076 \\
\hline
\end{tabular}

From the modal analysis of the various modes of vibration and vibration deformation can be seen, the natural frequency is above $80 \mathrm{~Hz}$, the laser cutting machine tool high overall natural frequency of the arm. But in the mode of order four, two or three arm, away from the machine bed distant arm rail vibration has great influence on the dynamic performance of the laser cutting machine, influence the machining accuracy of machine tool is serious, there is a weak link in the arm, must be to improve the structure of here, to improve the dynamic performance of the big arm. The same original appearance structure considering the laser cutting arm of the machine tool, in this case, at the end of the guiding rail and two ribs, the original rib plate and the thickness of rail is the same as that of the reinforcement of weak links in order to achieve the purpose .

The maximum modal natural frequency and vibration relative displacement of the improved large arms are shown in table 2:

Table 2 modal analysis results of the improved arm

\begin{tabular}{|c|c|c|}
\hline Modal order & natural frequency $/ \mathrm{Hz}$ & Maximum vibration relative position $/ \mathrm{mm}$ \\
\hline first order & 84.908 & 0.135206 \\
\hline second order & 114.22 & 0.127196 \\
\hline third order & 238.18 & 0.135648 \\
\hline fourth order & 335.62 & 0.156275 \\
\hline
\end{tabular}

It can be seen from the calculation results that the first and two order natural frequencies of the large arms are approximately the same with the original natural frequencies, and the other frequencies are improved. Through the improvement of the structure of the large arm, the maximum value of the relative displacement of each order vibration is reduced, and the amplitude is decreased, which improves the dynamic performance of the large arm. 


\section{Influence of laser focal position on laser cutting precision:}

The energy of laser focus is the highest, and the cutting efficiency is the highest. The relative position between the focal point and the material surface has great influence on the width of the slit and the shape of the side wall. Focus position with the amount of defocus, when the focus is on the surface of the material when the defocusing amount is zero, the above material is below the negative value of the focus to the vertical distance from the surface as shown in figure 3-2[9]. According to the material properties and the cutting requirements, in general, the amount of out of focus is zero or negative. In order to guarantee the cutting quality, the energy is the highest and the cutting efficiency is highest in the process of cutting. The relative position between the focus and the material surface has a great influence on the width of the slit and the appearance of the side wall of the cutting. Focus position with the amount of defocus, when the focus is on the surface of the material when the defocusing amount is zero, the above material is below the negative value of the focus to the vertical distance from the surface as shown in figure 9. According to the material properties and the cutting requirements, in general, the amount of out of focus is zero or negative. In order to ensure the quality of cutting, the focus is relatively fixed in the cutting process.

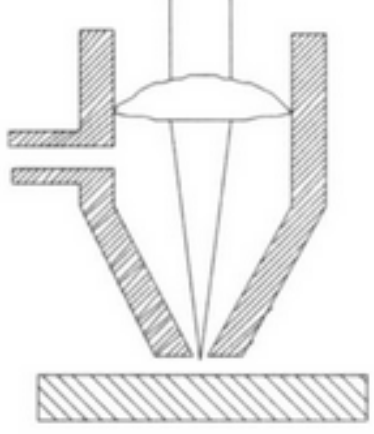

Positive focus

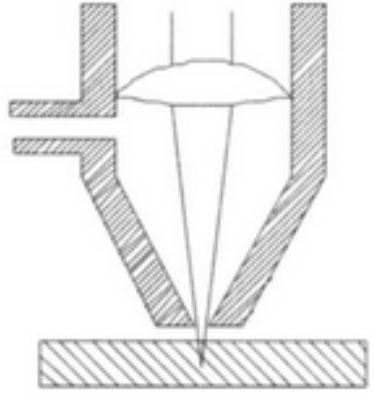

Negative coke

Fig. 9 focal position of laser cutting

The focal depth of the laser beam is $f_{d}$ and the focal length $f$ is approximately linear proportional relationship, the focal length $\mathrm{f}$ increases, the focal depth $f_{d}$ increases; the focal length $\mathrm{f}$ decreases, the focal depth $f_{d}$ becomes smaller. Beam through short focal length lens can get smaller spot diameter, is conducive to the cutting slit more narrow, the disadvantage is that the focal depth of more short $f_{d}$, is only suitable for cutting thin materials; long focal lens with long focal depth, can be used for cutting thicker material, but the beam radius increased. For cutting, generally want to focus on the spot diameter of $d$ is as small as possible, so that the power density can be improved, is conducive to the realization of high speed cutting, cutting width get smaller, but the focal spot diameter of $\mathrm{d}$ is too small, the depth of focus $f_{d}$ is too small, then it will be difficult to obtain good cutting surface verticality, so to maintain a certain depth of focus

\section{Influence of laser cutting speed on laser cutting precision:}

When the laser cutting speed change, time will change the laser and material, energy material in the unit area is changed, so the cutting speed will have a great influence on the precision of the laser cutting. When the cutting speed is low, the action time of the laser and material is longer, influence range is larger, the cutting seam around the material can easily be melting or vaporization, which easily lead to slit wide, rough cutting edge, the cutting quality is poor; with the increase of speed, when reaching a suitable range when the laser energy density can according to the cutting requirements will be completely removed to form a smooth uniform material, moderate width slit, get good cutting quality; when the speed increases, the laser energy density is reduced, not enough to completely remove the material, then the slit is narrow, but the cutting depth is unable to meet the requirements. When the velocity is increased to a limit value, the energy of the material is lower 
than that of the action threshold value, and can not be cut. As shown in Figure 3-3, when the laser repetition rate is $20 \mathrm{kHz}$, the cutting depth and cutting width of the single crystal silicon along with the cutting speed change relation diagram when nitrogen is used as an auxiliary gas. We can see that the cutting depth and the width of the cut are decreased with the increase of the cutting speed, which is consistent with the above analysis results ${ }^{[3]}$.

\section{The influence of different materials on the laser cutting precision:}

(1) if the melting point and the higher boiling point, it takes more energy to cut materials, which can remove the same energy of the material is less, so the kerf width will decrease;

(2) the thermal conductivity of materials is very good, easy to heat to get out, shorten the interaction in the time, the heat affected zone becomes small, slit width will decrease; poor thermal conductivity, the heat is not easy to get out, the melting area is limited to spot range, the width of the slit is limited ${ }^{[4]}$.

(3) the better absorption materials on the laser beam, more conducive to the effective use of energy of the laser beam, high quality cutting seam ${ }^{[5]}$.

(4) if the thickness of the material increases, the interaction time between the material and the laser will be increased, and the heat affected zone will become larger, so the width of the cut will be increased.

\section{Summary}

Based on the static analysis and modal analysis of the large arm of the laser cutting machine tool, the first four natural frequencies and modes of the large arm of the laser cutting machine tool are obtained. The dynamic performance of the large arm of the laser cutting machine tool can be directly analyzed by the vibration mode and the animation display, and the shortcomings of the large arm structure are found. Through the mode of the improved arm analysis, found the natural frequency of the arm improved the improved dynamic performance of the arm is improved effectively, provide a theoretical basis for the design and optimization of the structure and the cutting arm for the laser machine. At the same time through the analysis of laser focus effect on the precision of laser cutting, laser cutting speed and cutting precision of the influence of laser, different materials processed material influence on the precision of laser cutting, provides a way to study the specific processing requirements to the analysis of laser cutting machine. The characteristics of laser cutting machine must be compatible with the processing requirements, and can be applied to the economic and effective.

\section{Acknowledgement}

This research was financially supported by Research and practice of laser cutting machine adapting to the special processing requirements Project number: CXY201404

Dynamic analysis of three dimensional numerical control laser cutting machine Project number: B2015232;

Teaching reform and practice of material mechanics course based on the training of creative talents in the era of MOOCS Project number: 2015275;

\section{References}

[1] H.Akbulut. On optimization of a car rim using finite elementmethod[J].Finite Elements in Analysis and Design, 2003, 39: 433-443

[2]Wang bingxiu, Huo jindi. Study on the technology of laser cutting of stainless steel [J] Electric machining and die 2011.02,61-64

[3] Wu Yunxin, Deng Xishu Research on the theory and application technology of high precision 
laser cutting [D] Yantai University. 2009.04 .58-61

[4] Giovanni Tani, Luca Tomesani, Quality factors assessed by analytical modeling in laser cutting, Thin Solid Films, 2004, 453: 486 491.

[5] B.S. Yilbas, Laser cutting quality assessment and thermal efficiency analysis,

Journal of Materials Processing Technology, 2004, 155: 2106 2115 